\title{
Indians have narrower blood vessels - international expert
}

South African Indians (originally migrants from South Asia) have the highest rates of cardiovascular disease and diabetes in the country - and a fascinating contributor may be that their blood vessels are narrower than those of other race groups. Coronary angiograms and postmortem studies reveal this little known fact, according to Dr Alexander T Cohen, honorary consultant vascular physician at Kings College Hospital in London, who cautioned that the high diabetes rates were primarily responsible for the cardiovascular elevation.

This emerged in an interview with Cohen who recently completed a lecture tour of South Africa updating doctors, pharmacists and nurses on a ground-breaking new oral therapy for venous thrombo-embolism (VTE). Cohen is also an epidemiologist involved in clinical work, designing, managing and analysing clinical trials from phase 1 to $1 \mathrm{~V}$, and both chairs and serves on several international steering committees for multicentre trials, and epidemiological and pharmaco-economic studies. The nugget about lower-calibre blood vessels in Indians emerged when he was asked his opinion on why the highest rates of heart and blood vessel disease in South Africa occur in this community (followed by the coloured community, with the white and black communities having the lowest and most similar rates).

He also put the dramatically differing patterns between the white and black local populations (in spite of their having similar

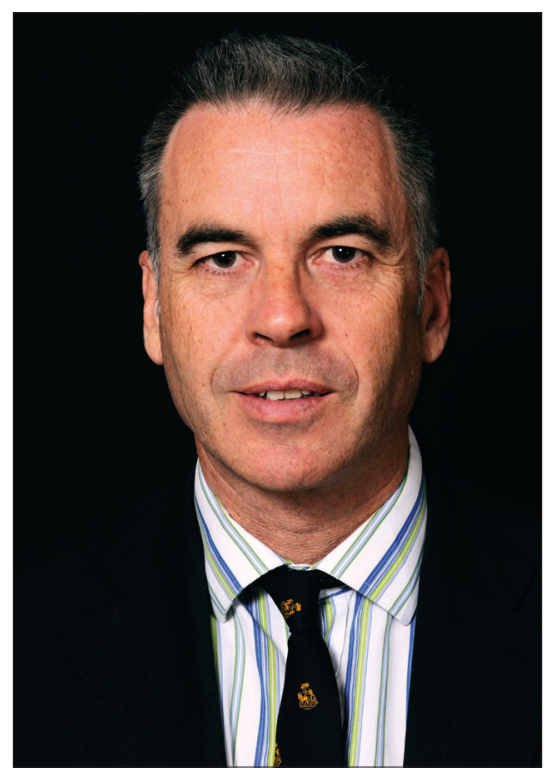

Dr Alexander $T$ Cohen, honorary consultant vascular physician at Kings College Hospital in London.

rates of disease) down to research skewed by healthcare inequities. Seemingly, the white population predominantly has a pattern of deaths due to heart attacks, while the black population predominantly has a pattern of deaths due to stroke, heart muscle disease and heart disease due to high blood pressure. 'What you may possibly be seeing in some centres in South Africa is that a black person comes with a swollen leg and the doctor is not suspecting venous thrombosis and hence says take an aspirin or anti-inflammatory and they go. This is because the level of clinical suspicion or pretest probability may be low in this racial group. However, this is not borne out by research in African Americans where VTE rates are similar or higher than whites. In contrast, if a white person comes in and is seen in private the clinical suspicion is higher, the threshold for investigation is likely to be lower and hence they get a leg scan and the DVT is diagnosed, or in the case of respiratory symptoms they are sent for a lung scan.' His comments were greeted with some alarm by local public sector vascular surgeons and haematologists afraid that they would appear uncaring along racial lines, but Cohen was referring to the overall healthcare system (split between medically insured and medically uninsured) where glaring racial inequities in access and treatment persist, particularly at primary healthcare level from where public sector patients are referred upwards.

Referring to the Indian vascular anomaly, he said Indians were genetically Caucasian and varied little from the Western European population. Indians, Pakistanis, Bangladeshis and Sri-Lankans had the highest rates of diabetes and lipid abnormalities, and high rates of cardiovascular disease (in particular arterial and venous disease). 'Interestingly when you do the investigations, the width of their vessels is somewhat narrower. It's potentially a big problem. You see this in coronary angiograms. The blood vessels are just not as wide. The things that make it different in the rate of venous thrombosis are much broader than what makes it different in arterial thrombosis. 
In venous clots (deep vein thrombosis (DVT)) however, it's a completely different set of risk factors', he added. The aforementioned factors were present but very weak. Rather, immobilisation (especially after a stroke), all types of surgery, infections, heart failure, respiratory failure, cancer, rheumatological disorders and oral contraceptives came to the fore with DVT. If all of these were eliminated, then blood tests often revealed a congenital predisposition, while flying for more than four hours was a significant risk.

\section{Doctors missing VTE - Cohen}

Cohen believes research showing that heart and blood vessel disease claimed 195 South African lives per day between 1997 and 2004 actually underestimates the true situation. This is because for every blood clot diagnosis prior to death another two are found on autopsy that were not suspected. He believes that the associated morbidity and mortality are 'significantly elevated' because of HIV/AIDS and tuberculosis. Cohen said blood clots were easily and often misdiagnosed. He said the chronic nature of VTE and its complications, such as post-thrombotic syndrome and pulmonary hypertension, generated a considerable health burden. In the absence of thromboprophylaxis, DVT developed in $40-60 \%$ of patients undergoing total knee replacement or total hip replacement and in 10 - $40 \%$ of medical and general surgery patients. After coronary heart disease and stroke, VTE was the third most common cardiovascular disease, with more than a third of cases representing recurrent VTE. The number of VTE deaths in Europe was three times greater than the combined number of deaths from breast cancer, AIDS and road traffic accidents. A VTE impact assessment group in Europe estimating the total mortality burden of VTE in France, Germany, Italy, Spain, Sweden and the UK (12\% of all deaths), found that $59 \%$ occurred after undiagnosed pulmonary embolism. Intrinsic risk factors included gender, age and malignancy, while extrinsic risk factors included recent surgery or trauma.

\section{New oral therapy a treatment boon}

Cohen said the new oral therapy for VTE, rivaroxaban (in use for nearly six months in the UK, a year in Canada and Germany and nearly five months in Australia), was ground breaking. Whereas injected heparins (like enoxaparin and dalteparin) and oral warfarin resulted in an increased chance of cerebral bleeding, rivaroxaban was both safer and reduced the incidence of all major and critical bleeding. Rivaroxaban removed the need for heparin injections, the regular blood tests and the monitoring needed with the traditional duo of drug treatments; it was also less expensive and vastly easier for the physician to manage. A big advantage was that patients did not have to have their warfarin levels monitored by INR on a regular basis. The Federal Drug Administration (FDA) in the USA recently approved the drug which Cohen predicted would create 'a sea of change in the way we practice.

\section{Chris Bateman}

chrisb@hmpg.co.za

S Afr Med J 2012;102(12):906-907.

DOI:10.7196/SAMJ.6465 